\title{
Antibiotics used in the ambulatory management of acute COPD exacerbations
}

\author{
Marie-France \\ Beauchesne $e^{1,2,3}$ \\ Marcel Julien 2,4 \\ Louis-André Julien ${ }^{2,4}$ \\ Dominique Piquette ${ }^{4}$ \\ Amélie Forget ${ }^{2}$ \\ Manon Labrecque ${ }^{2,4}$ \\ Lucie Blais ${ }^{1,2,3}$ \\ 'Faculty of Pharmacy, ${ }^{4}$ Faculty of \\ Medicine, University of Montréal, \\ Montréal, Québec, Canada; ${ }^{2}$ Hôpital \\ du Sacré-Coeur de Montréal, Gouin \\ Ouest, Montréal, Québec, Canada; \\ ${ }^{3}$ Endowment Pharmaceutical Chair \\ AstraZeneca in Respiratory Health, \\ Montréal, Québec, Canada
}

Correspondence: Marie-France Beauchesne

Pharmacy, Hôpital du Sacré-Cœur de Montréal, 5400 boul. Gouin ouest, Montreal, Quebec, H4J IC5, Canada

$\mathrm{Tel}+\mathrm{I} 51433822222666$

Fax + I 5143383200

Email marie-france.

beauchesne@umontreal.ca
Study objectives: This study was conducted to describe the different antibiotics that are used in the home management of chronic obstructive pulmonary disease (COPD) exacerbations and to estimate the failure rates following the initiation of the antibiotic.

Methods: A cohort study was conducted. Patients enrolled in a COPD home management program were included in the analysis. Failure rates were defined as an additional prescription of an antibiotic, an emergency room visit, or a hospitalization for a COPD exacerbation in the 30 days following the initiation of the antibiotic.

Results: A total of 1180 episodes of antibiotic treatment were analyzed. Overall, 348 episodes led to a failure $(29.5 \%)$. The most frequently used antibiotics were cefuroxime $(45.9 \%)$ and ciprofloxacin $(21.1 \%)$.

Conclusion: This project demonstrates that a wide range of antibiotics were prescribed to our population of COPD patients with a moderate to severe form of the disease. Many treatment failures (about 30\%) occurred in the 30-day period following the initiation of the home therapy with an antibiotic. Clinicians should be aware of this high failure rate when managing mild exacerbations of COPD at home.

Keywords: acute exacerbation of COPD, antibiotics, COPD

\section{Introduction}

Several trials have demonstrated that antibiotics are beneficial over placebo for the treatment of acute exacerbations of chronic obstructive pulmonary disease (COPD) (Anthonisen et al 1987; Saint et al 1995; Ram et al 2006). COPD guidelines recommend the use of antibiotics when clinical signs of infection are apparent; for example, increased sputum volume and purulence, and/or fever (GOLD 2007). Antimicrobial choices can be based upon the local patterns of antibiotic sensitivity among the most common responsible pathogens (GOLD 2007). Canadian guidelines recommend the use of amoxicillin, doxycycline, trimethoprim/sulfamethoxazole, second or third-generation cephalosporins, or extended spectrum macrolides in the absence of risk factors, whereas fluoroquinolones or beta-lactam/beta-lactamase inhibitor (amoxicillin/clavulanic acid) are suggested for patients who have an forced expiratory volume in one second $\left(\mathrm{FEV}_{1}\right)$ lower than $50 \%$ of the predicted, four or more COPD exacerbations per year, ischemic heart disease, home oxygen use, chronic use of oral corticosteroids, and/or antibiotherapy taken in the previous three months (O'Donnell et al 2007). The authors do however, specify that this is a level $3 \mathrm{c}$ recommendation ("consensus from expert groups based on clinical experience and poor evidence to support a recommendation for or against use"). Furthermore, it is also specified that antibiotics for patients with no risk factors when combined with prednisone may suffice (O’Donnell et al 2007).

Most patients included in our COPD home management program have the risk factors associated with the use of second line antimicrobial agents (ie, for patients with 
risk factors), specified in the Canadian COPD guidelines, but various antibiotics, including first-line agents, are used. Therefore, we sought to describe the type of antibiotics that are used by COPD patients included in our home management program and to estimate the failure rate associated with this therapy.

\section{Methods}

We conducted a retrospective cohort study to describe the different antibiotics that are used for COPD patients included in our home management program, and estimate the treatment failure rates. All patients participating in the COPD home-management program of l'Hôpital du Sacré-Coeur de Montréal (HSCM) were included in the analysis if they had follow-up data for at least 12 months. This program follows patients for whom COPD represents a chronic disease that has an important impact on their quality of life and who are at risk of severe exacerbations; for example, have had previous exacerbations requiring acute care. Patient charts updated at least every month by doctors, nurses, and pharmacists were used for data collection. Cohort entry corresponded to inclusion in the program (started in 1995) and follow-up until exclusion from the program, death, or November 31 st, 2004, whichever occurred first. Antibiotics were initiated at home for these patients when they reported at least two of the following criteria: dyspnea, increased sputum production, increased sputum volume (Anthonisen et al 1987). The selection of an appropriate antibiotic for the patient's action plan is at the physician's discretion and is not based upon prespecified criteria with the exception of drug allergy or intolerance. Usually, the action plan (antibiotic) is prescribed for a year and rotation from one antibiotic to another over the course of the year to treat subsequent exacerbations is not done unless treatment fails. Microbiologic data is not usually available to select the antibiotic; patients who begin an exacerbation are managed by telephone communication between the nurse and the patient. Usual treatment duration is of 10 days (with the exception of azithromycin which in most cases is prescribed for 5 days). An intravenous antibiotic regimen and a concomitant use of two antibiotics were excluded from the analysis, since these cases are managed differently (usual protocol is not followed for these cases at our program). Patients included in this program are followed by one of the respiratory physicians at HSCM with meetings once or twice a year at the outpatient clinic.

Data collected included antibiotic treatments (number and agents used), number of emergency room visits for a COPD exacerbation, number of hospitalizations for a
COPD exacerbation, use of oral and inhaled corticosteroids, use of bronchodilators (inhaled short and long-acting bronchodilators, theophylline), $\mathrm{FEV}_{1}$, level of dyspnea (based on the cardiac rhythm management dyspnea index; scale 1-5, a higher score implies a higher level of dyspnea), co-morbidities, age, sex, smoking status, home oxygen use, hypercapnia, and level of autonomy (scale 1-6, a higher score implies a better level of autonomy).

The main outcome was the type of antimicrobial agents prescribed to the patients in the cohort. The secondary outcome was treatment failure in the 30 days following the initiation of the antibiotic. Treatment failure was considered if one of the following events occurred in the 30-day period following the initiation of the antibiotic: prescription of the same antibiotic for home management once the initial treatment has been completed; use of a different antimicrobial agent for home management of a COPD exacerbation (change in the antibiotic initially prescribed before treatment completion or upon treatment completion); emergency room visit for a COPD exacerbation (as soon as 1 day following treatment initiation); and hospitalization for a COPD exacerbation (as soon as 1 day following treatment initiation). These were described separately and then, treatment failure was assessed based on the occurrence of one of these four events; if more than one occurred in the 30-day period, only one treatment failure was calculated. Furthermore, a delay of at least 30 days between two exacerbations in the same patient was mandatory to consider the occurrence of a new COPD exacerbation requiring antibiotic therapy. The conduct of this project was approved by the ethics committee of our institution.

\section{Statistical analysis}

Descriptive statistics - proportions and standard deviation were calculated to describe the type of antibiotics prescribed.

\section{Results}

The initial cohort included 2288 episodes of oral antibiotic treatments received by 152 COPD patients included in the home management program between 1995 and 2004. We excluded 972 antibiotic treatments that were preceded in the 30 days by a COPD exacerbation (hospitalization, emergency room visit, another antibiotic therapy), and 136 episodes for which complete clinical data of the patients were not available. Therefore, a total of 1180 episodes of antibiotic treatment received by 152 patients were analyzed. The distribution of the characteristics of the patients at cohort entry 
and antibiotic choices are found in Table 1 and Table 2. All patients included in our cohort had an $\mathrm{FEV}_{1}$ lower than $50 \%$ at cohort entry, and therefore were in the category "with risk factors" based on the Canadian guidelines. The most frequently used antibiotics were cefuroxime (45.9\%) and ciprofloxacin (21.1\%). A total 473 of the 1180 antibiotic treatments $(40.0 \%)$ were combined with prednisone.

Overall, 348 of the 1180 episodes led to a treatment failure (29.5\%), including 103 hospitalizations for a COPD exacerbation, 29 emergency room visits, 44 repeated treatments with the same antibiotic, and 172 repeated treatments with a different antibiotic (Table 3). The average time between

Table I Characteristics of the patients at their entry in the COPD home management program

\begin{tabular}{|c|c|}
\hline Characteristics $(n=152)$ & $\mathbf{N}(\%)$ \\
\hline Gender: Male & $76(50.0)$ \\
\hline \multicolumn{2}{|l|}{ Mean age: } \\
\hline 49-64 years old & $31(20.4)$ \\
\hline $65-74$ years old & $60(39.5)$ \\
\hline$\geq 75$ years old & $61(40.1)$ \\
\hline \multicolumn{2}{|l|}{ BMI: } \\
\hline$<20$ & $37(24.3)$ \\
\hline $20-29$ & $92(60.5)$ \\
\hline$\geq 30$ & $23(15.1)$ \\
\hline \multicolumn{2}{|l|}{ Smoking status: } \\
\hline Past history of smoking & $120(79.0)$ \\
\hline Smoker & $26(17.1)$ \\
\hline No history of smoking & $6(4.0)$ \\
\hline \multicolumn{2}{|l|}{ Number of co-morbiditites: } \\
\hline$<2$ & $88(57.9)$ \\
\hline$\geq 2$ & $64(42.1)$ \\
\hline \multicolumn{2}{|l|}{ Dyspnea index (scale of I-5) } \\
\hline $1-2$ & $19(12.5)$ \\
\hline 3 & $29(19.1)$ \\
\hline $4-5$ & $104(68.4)$ \\
\hline \multicolumn{2}{|l|}{ Level of autonomy (scale I-6) } \\
\hline $1-3$ & $78(55.3)$ \\
\hline $4-6$ & $63(44.7)$ \\
\hline Home oxygen & $70(46.4)$ \\
\hline (albuterol and ipratropium) & $152(100)$ \\
\hline Use of a long-acting beta-2 agonist & $87(57.2)$ \\
\hline Use of an inhaled corticosteroid & $132(86.8)$ \\
\hline Use of theophylline & $56(36.8)$ \\
\hline Continuous use of oral corticosteroids & $22(14.6)$ \\
\hline \multirow[t]{2}{*}{$\mathrm{FEV}_{1}$} & Mean $( \pm S D)$ \\
\hline & $32.3 \%$ predicted $( \pm 9.1)$ \\
\hline Annual rate of exacerbations & $2.9( \pm 2.3)$ \\
\hline
\end{tabular}

Notes: The mean number of pack-years of smoking was unknown; Based on the CRM dyspnea index:a higher score implies a higher level of dyspnea; Questionnaire developed for our program (not validated): a higher score implies a better level of autonomy. The six-minute walk distance is unknown.

Abbreviations: $\mathrm{BMI}$, body mass index; $\mathrm{FEV}_{1}$, forced expiratory volume in one second $\mathrm{SD}$, standard deviation.
Table 2 Antibiotics used to treat acute exacerbations of COPD at home

\begin{tabular}{ll}
\hline Antibiotics used ( $\mathbf{n}$ = I I 80 treatments) & $\mathbf{N}(\%)$ \\
\hline Cefuroxime & $542(45.9)$ \\
Ciprofloxacin & $249(2 \mathrm{I} . \mathbf{1})$ \\
Levofloxacin & $73(6.2)$ \\
Azithromycin & $56(4.8)$ \\
Amoxicillin & $49(4.2)$ \\
Moxifloxacin & $48(4.1)$ \\
Trimethoprim-sulfamethoxazole & 4 I (3.5) \\
Gatifloxacin & $24(2.0)$ \\
Amoxicillin-clavulanic acid & $20(1.7)$ \\
Others (ampicillin, cephalexin, cefaclor, cefixime, & $37(3.1)$ \\
cefprozil, clindamycin, cloxacillin, & \\
erythromycin, ofloxacin, penicillin, & \\
pivampicillin, trovafloxacin) & \\
\hline
\end{tabular}

the initiation of the antibiotic and the treatment failure was 15.3 days (median of 14 days).

A total of 418 of the 1180 treatments prescribed (35.4\%) were antibiotics recommended for patients with risk factors according to the Canadian COPD guidelines (ie, amoxicillin/clavulanic acid or fluroquinolones). The failure rates associated with the use of an antibiotic for patients with risk factors was of $32.8 \%$ while it was of $27.7 \%$ for antibiotics in the category of patients without risk factors. The risk of having a treatment failure was slightly higher when an antibiotic for patients with risk factors was prescribed (data not shown, available upon request).

\section{Discussion}

Overall, our failure rate was greater than the ones reported in the literature that vary from $12 \%$ to $21 \%$ (Adams et al 2000; Dewan et al 2000; Miravitlles et al 2001). Potential explanations for this difference are our study's inclusion of patients with a more severe form of the disease, and the use of a different definition of failure (Adams et al 2000; Dewan et al 2000; Miravitlles et al 2001). Furthermore, about 65\% of patients received an antibiotic for patients with no risk factors, while they were considered at risk based on their characteristics (such as $\mathrm{FEV}_{1}$ ). A deficiency in guideline conformity and under use with regard to COPD treatment has been previously reported (Jans et al 1998; Roche et al 2001; Choi et al 2004; Brand et al 2005; Fritsch et al 2005; Boulet et al 2006; Glaab et al 2006a, 2006b). However, antibiotic treatment recommendations in the Canadian COPD guidelines are not based on a high level of evidence.

The main strength of our study is that we collected data from a real clinical setting. But our results cannot be generalized to all COPD patients since the patients included 
Table 3 Description of treatment failure ( $N=348$ failures for I I 80 episodes of treatment)

\begin{tabular}{ll}
\hline Type of failure & N (\%) \\
\hline Emergency room visit & $29(8.3)$ \\
Hospitalization & $100(28.7)$ \\
Re-prescription of the same antibiotic & $44(12.6)$ \\
Re-prescription of the same antibiotic followed & $\mathrm{I}(0.3)$ \\
by a hospitalization & \\
$\begin{array}{l}\text { Prescription of a different antibiotic } \\
\text { Prescription of a different antibiotic followed } \\
\text { by a hospitalization }\end{array}$ & $2(0.6)$ \\
\hline
\end{tabular}

in our program have a severe form of the disease. We attempted to compare failure rates between different classes of antibiotic (data not shown) by performing a Chi-square test and a Poisson regression analysis, but our study design had many limitations such as lack of power and the absence of randomization. In fact, patients who received large spectrum antibiotics had a higher risk of failure even after adjusting our results for confounding variables. This suggests that patients who received large spectrum of antibiotics were the ones with the most severe disease (ie, higher risk of failure) and we could not completely exclude the possibility of residual confounding of disease severity in the analysis of our results.

In conclusion, a wide range of antibiotics are prescribed to our patients with moderate to severe COPD to treat mild exacerbations at home. Overall, the initiation at home of an antibiotic for an acute exacerbation of COPD failed to prevent the use of another antibiotic treatment, an emergency room visit, or a hospitalization in $30 \%$ of the cases. Results of this study should alert the clinician to the high risk of treatment failure when managing mild exacerbations of COPD at home in patients with severe disease. Furthermore, our results support the implementation of strategies to better promote the application of clinical practice guidelines for the management of chronic diseases.

\section{Disclosure}

This study was funded by Bayer Canada Inc. Marie-France Beauchesne has participated in continuing education programs sponsored by the pharmaceutical industry, such as GSK, BI, Pfizer, and AstraZeneca. She has also received grants from Merck Frosst, GSK, and AstraZeneca for some of her research projects. Lucie Blais is a recipient of a New Investigator Salary Support Grant from the Canadian Institutes for Health Research (CIHR). She has received support from AstraZeneca, GSK, and Amgen for some of her research projects. Manon Labrecque has participated in CE programs or advisory boards for AstraZeneca, AltanaPharma, and GSK. She has also received grants from Merck Frosst, GSK, and AstraZeneca for some of her research projects. Marcel Julien has participated in an advisory board sponsored by BI.

\section{References}

Adams SG, Melo J, Luther M, et al. 2000. Antibiotics are associated with lower relapse rates in outpatients with acute exacerbations of COPD. Chest, 117:1345-52.

Anthonisen NR, Manfreda J, Waren CPW, et al. 1987. Antibiotic therapy of acute exacerbations of chronic obstructive pulmonary disease. Ann Intern Med, 106:196-204.

Boulet L, Becker A, Bowie D, et al. 2006. Implementing practice guidelines: a workshop on guidelines dissemination and implementation with a focus on asthma and COPD. Can Respir J, 13(Suppl A):5-47.

Brand C, Landgren F, Hutchinson A, et al. 2005. Clinical practice guidelines: barriers to durability after effective early implementation. Intern Med J, 35:162-9.

Choi PP, Day A, Etchells E. 2004. Gaps in the care of patients admitted to hospital with an exacerbation of chronic obstructive pulmonary disease. CMAJ, 170:1409-13.

Dewan NA, Rafique S, Kanwar B, et al. 2000. Acute exacerbation of COPD. Factors associated with poor treatment outcome. Chest, 117:662-71.

Fritsch K, Jacot ML, Klarer A, et al. 2005. Adherence to the Swiss guidelines for management of COPD: experience of a Swiss teaching hospital. Swiss Med Wkly, 135:116-21.

Glaab T, Banik N, Trautmann M, et al. 2006. Guideline-conformity of outpatient COPD management by pneumologists. Pneumologie, 60:395-400.

Glaab T, Banik N, Singer C, et al. 2006. Guideline conformance for outpatient management of COPD in Germany. Dtsch Med Wochenschr, 21:1203-8.

[GOLD] Rabe KF, Hurd S, Anzueto A, et al; Global Initiative for Chronic Obstructive Lung Disease. 2007. Global strategy for the diagnosis, management, and prevention of chronic obstructive pulmonary disease: GOLD executive summary. Am J Respir Crit Care Med, 176:532-55.

Jans MP, Schellevis FG, van Hensbergen W, et al. 1998. Management of asthma and COPD patients: feasibility of the application of guidelines in general practice. Int J Qual Health Care, 10:27-34.

Miravitlles M, Murio C, Guerrero T, et al. 2001. Factors associated with relapse after ambulatory treatment of acute exacerbations of chronic bronchitis. Eur Respir J, 17:928-33.

O'Donnell DE, Aaron S, Bourbeau J, et al. 2007. Canadian Thoracic Society recommendations for management of chronic obstructive pulmonary disease-2007 update. Can Respir J, 14:5B-32B.

Ram FSF, Rodriguez-Roisin R, Granados-Navarrete A, et al. 2006. Antibiotics for exacerbations of chronic obstructive pulmonary disease. Cochrane Database Syst Rev, 2:CD004403.

Roche N, Lepage T, Bourcereau J, et al. 2001. Guidelines versus clinical practice in the treatment of chronic obstructive pulmonary disease. Eur Respir J, 18:903-8.

Saint S, Bent S, Vittinghoff E, et al. 1995. Antibiotics in chronic obstructive pulmonary disease exacerbations: a meta-analysis. JAMA, 273:957-60. 